\title{
Narrative Centrality and Negative Affectivity: Independent and Interactive Contributors to Stress Reactions
}

\author{
David C. Rubin \\ Duke University and Aarhus University
}

\author{
Adriel Boals \\ University of North Texas
}

\author{
Rick H. Hoyle \\ Duke University
}

\begin{abstract}
Reactions to stressful negative events have long been studied using approaches based on either the narrative interpretation of the event or the traits of the individual. Here, we integrate these 2 approaches by using individual-differences measures of both the narrative interpretation of the stressful event as central to one's life and the personality characteristic of negative affectivity. We show that they each have independent contributions to stress reactions and that high levels on both produce greater than additive effects. The effects on posttraumatic stress symptoms are substantial for both undergraduates (Study 1 , $n=2,296$; Study 3, $n=488$ ) and veterans (Study 2, $n=104$ ), with mean levels for participants low on both measures near floor on posttraumatic stress symptoms and those high on both measures scoring at or above diagnostic thresholds. Study 3 included 3 measures of narrative centrality and 3 of negative affectivity to demonstrate that the effects were not limited to a single measure. In Study $4(n=987)$, measures associated with symptoms of posttraumatic stress correlated substantially with either measures of narrative centrality or measures of negative affectivity. The concepts of narrative centrality and negative affectivity and the results are consistent with findings from clinical populations using similar measures and with current approaches to therapy. In broad nonclinical populations, such as those used here, the results suggest that we might be able to substantially increase our ability to account for the severity of stress response by including both concepts.
\end{abstract}

Keywords: autobiographical memory, narrative, neuroticism, identity, posttraumatic stress disorder

Narrative is the means used to express events. The psychological study of narrative is extensive and diverse (e.g., Baumeister \& Newman, 1994; Bruner, 1986; Fivush \& Hayden, 2003; Freeman, 1993; McAdams, 1996; Rubin, 2006; Spence, 1982). In the study of personality, life stories are the foundation of major theoretical approaches (for reviews, see McAdams, 1996; McAdams, Josselson, \& Lieblich, 2006; McAdams \& McLean, 2013). In clinical psychology, early therapies were often based on understanding the narratives told by patients. This continues in modern times in the form of psychotherapies that seek to modify or reinterpret narratives. Changing the narratives of memories of negative or stressful events to have less negative interpretations and implications is a

This article was published Online First December 2, 2013.

David C. Rubin, Department of Psychology and Neuroscience, Duke University, and Center on Autobiographical Memory Research, Aarhus University, Aarhus, Denmark; Adriel Boals, Department of Psychology, University of North Texas; Rick H. Hoyle, Department of Psychology and Neuroscience, Duke University.

We wish to thank Dorthe Berntsen, Christin Ogle, and Dorthe Thomsen for comments and National Institute of Mental Health Grant R01 MH066079 and Danish National Research Foundation Grant DNRF93 for funding.

Correspondence concerning this article should be addressed to David C. Rubin, Department of Psychology and Neuroscience, Box 90086, Durham, NC 27708-0086. E-mail: david.rubin@ duke.edu common strategy for comforting people in nonclinical settings and for reducing symptoms in clinical settings (e.g., Bryant, 2011; Hembree \& Foa, 2010). In acute and posttraumatic stress disorder (PTSD), the narrative takes on an added role in that a narrative is used to evaluate the criteria and symptoms needed to diagnose PTSD. Here, we measure narrative centrality, the extent to which a stressful event, which itself may be remembered and told as a narrative, is central to a person's overall narrative identity.

A second and very different behavioral approach to understanding reactions to stressful events is the measurement of individual differences, including personality tests. In contrast to narratives of events, personality traits are seen as a relatively stable attribute of a person, which is expressed in a wide range of cognition, emotion, and behavior rather than being focused on a single event. In the individual-differences approach used here, personality has been divided into a small number of traits, usually five or fewer (Digman, 1990; Malouff, Thorsteinsson, \& Schutte, 2005), or two to three broader factors (Tellegen, 1985; Watson \& Clark, 1984).

Narratives of single events and personality traits are fundamentally different. Narrative "strives to put its timeless miracles into the particulars of experience, and to locate the experience in time and place" (Bruner, 1986, p. 13). It is not a general trait of an individual; the narrative of a particular event can be changed without changing narratives of other events or behaviors. Several types of therapies shown to reduce PTSD symptoms, such as cognitive processing therapy (Resick \& Schnicke, 1992), focus on 
changing the narrative interpretation of the traumatic event. In contrast, personality, particularly high-order dimensions such as negative affectivity, is nonspecific and generally stable across time and situations (Caspi, Roberts, \& Shiner, 2005), though it is not fixed and changes over the life span and with life circumstances (e.g., Neyer \& Lehnart, 2007; Roberts, Walton, \& Viechtbauer, 2006).

In addition, measures of personality and narrative differ in the time course of how they are affected by traumatic events. Personality is assumed to be relatively stable in adults and should change less over time than the narrative interpretation of an event. Previous studies have indicated that negative affectivity, frequently measured by the personality trait neuroticism, is not affected by negative events such as moving from one's parents' home, divorce, and unemployment (Costa, Herbst, McCrae, \& Siegler, 2000; Specht, Egloff, \& Schmukle, 2011; Sutin, Costa, Wethington, \& Eaton, 2010) or more traumatic events encountered in middle adulthood (Ogle, Rubin, \& Siegler, in press). In contrast, the time course of narrative measures is more dynamic. The interpretation of whether a negative event is central to identity, resolved, or related to positive growth cannot exist until the event occurs, and then, its interpretation should change over time in a healthy individual. That is, the narrative should depend not only on information, attitudes, and skills obtained before the trauma but also on changes in them and the environment during and after the trauma.

Not only are measures of narratives of single events and personality tests different, they have not been included in the most commonly studied factors correlated with PTSD. For instance, an extensive meta-analysis (Brewin, Andrews, \& Valentine, 2000) used the following categories: gender, younger age, low socioeconomic status, lack of education, low intelligence, race, psychiatric history, childhood abuse, other previous trauma, other adverse childhood factors, family psychiatric history, trauma severity, lack of social support, and life stress. A similarly comprehensive metaanalysis (Ozer, Best, Lipsey, \& Weiss, 2003) used prior trauma, prior adjustment, family history of psychopathology, perceived life threat, perceived support, peritraumatic emotions, and peritraumatic dissociation. Neither included the two concepts investigated here. Moreover, as is reviewed later, measures of narrative centrality and negative affectivity correlate highly with PTSD symptom severity, even in multiple regressions with many other measures included and even in prospective studies (Boelen, 2012; Rubin, Berntsen, \& Bohni, 2008).

\section{The Current Studies}

We investigate and combine measures from two distinct behavioral approaches, which contribute broadly to our understanding of long-term reactions to negative stressful events: narrative and personality. Within narrative, we focus on measures of narrative centrality, and within personality, we focus on measures of negative affectivity. There are three main reasons for choosing these two behavioral approaches and these particular measures. First, narrative centrality and negative affectivity are conceptually distinct measures with different time courses. The narrative centrality of an event can be changed without necessarily changing the narrative centrality of other events, whereas negative affectivity affects a broad range of behaviors and thus changes to it would affect behavior broadly. Narrative centrality can exist only after the event occurs, whereas negative affectivity is seen as developing over the life span. Second, narrative centrality and negative affectivity both correlate highly with measures of symptoms of stress to negative events, arguably higher than any other individualdifferences measures. Moreover, consistent with their conceptual differences, their contributions to stress reactions are not highly correlated, suggesting that they could be addressed separately to reduce symptom severity. Third, though well studied individually, neither has been included in meta-analyses of stress reactions in the trauma literature or otherwise integrated into the literature on stress reactions. In particular, narrative centrality was initially introduced to counter the idea present in some views of PTSD that not integrating trauma memories into one's life story could increase clinical symptoms. This view predicts that narrative centrality would correlate negatively with PTSD symptom severity. However, narrative centrality has consistently had substantial positive correlations with clinical symptoms in clinical disorders including PTSD, depression, and chronic pain (e.g., Perri \& Keefe, 2008; Pinto-Gouveia \& Matos, 2011; Rubin, Dennis, \& Beckham, 2011), and so, it is time to move beyond the initial motivation and examine its effects more systematically.

Here, we use three measures to focus on the centrality of a narrative of a single negative event to a person's life story and narrative identity (McAdams \& McLean, 2013). We chose these measures because they were the only measures we could find. They are the Centrality of Event Scale (CES; Berntsen \& Rubin, 2006), the Closure Scale (Beike \& Wirth-Beaumont, 2005), and the Posttraumatic Growth Inventory (PTGI; Tedeschi \& Calhoun, 1996). Although the three measures each come from a different theoretical tradition, they each assess an aspect of an individual's interpretation of how central the narrative of an autobiographical memory about a negative event is to his or her life story and narrative identity, rather than objective reactions or objective severity (see Rubin \& Feeling, 2013). In addition, they each correlate highly with PTSD symptom severity.

The CES measures the extent to which individuals construe a stressful or traumatic event as a central component of their narrative identity and sense of self. Components of this measure include the extent to which an event colors the way individuals interpret new experiences, signals a major turning point in their life story, and has become a core component of their narrative identity. The CES has strong empirical support in correlating with PTSD and symptoms of posttraumatic stress across a variety of participant samples and trauma types (e.g., Boals \& Schuettler, 2011; Boelen, 2009; Brown, Antonius, Kramer, Root, \& Hirst, 2010; Newby \& Moulds, 2011; Pinto-Gouveia \& Matos, 2011; Robinaugh \& McNally, 2010; Schuettler \& Boals, 2011). Moreover, past studies have typically found that in multiple regression analyses, the CES accounts for variance in PTSD symptoms, even after controlling for a number of other known risk factors of PTSD symptoms, such as anxiety, depression, dissociation, and characteristics of the event (Berntsen \& Rubin, 2006; Boals, 2010; Brown et al., 2010; Schuettler \& Boals, 2011). Of direct relevance here, the CES accounts for variance even after negative affectivity is included (Berntsen \& Rubin, 2007; Boelen, 2012; Rubin, Boals, \& Berntsen, 2008; Rubin et al., 2011). 
The Closure Scale measures the extent to which individuals believe they have attained psychological closure and resolution concerning a negative experience. It is an important component of individuals' narrative of a negative event and its impact. When such resolution has been obtained, the negative event is less likely to be central to narrative identity. Memories that lack closure have high levels of intense negative emotional details (Beike \& WirthBeaumont, 2005; Crawley, 2010), are more likely to involve attempts at meaning making (Boals, Banks, Hathaway, \& Schuettler, 2011), and have a directive effect on behavior (Beike, Adams, \& Naufel, 2010).

The PTGI was originally designed to measure positive changes in a person's worldview and social relations as caused by a traumatic event (Tedeschi \& Calhoun, 1996). It includes items that measure the extent to which the trauma experience has affected the appreciation of life, spiritual change, personal growth, ability to relate to others, and new possibilities. However, the PTGI measures perceived growth rather than actual growth (Coyne \& Tennen, 2010; Frazier et al., 2009), which is preferable for the purpose of measuring narrative centrality. Events that are central to identity and for which there is not yet closure have the potential to be seen as resulting in posttraumatic growth (Boals \& Schuettler, 2011; Schuettler \& Boals, 2011). Higher ratings on the PTGI are viewed as positive by the participant even though those higher ratings are often associated with more symptoms of posttraumatic stress.

With regard to measures of personality, following the trait dimensional diagnostic system for personality disorder in the Diagnostic and Statistical Manual of Mental Disorders (5th ed., American Psychiatric Association, 2013), we use the term negative affectivity to refer to the broader concept that encompasses the trait of neuroticism and portions of extraversion and agreeableness. Two of the four measures of negative affectivity we use are based on standard, established approaches to measuring negative affectivity broadly construed: Neuroticism $(\mathrm{N})$ from the Big Five Inventory (BFI; John, Donahue, \& Kentle, 1991) and Negative Affect (NA) from the Positive and Negative Affect Schedule (PANAS; Watson, Clark, \& Tellegen, 1988). The third, the Affect Intensity Measure-Negative Intensity (AIM-NI), comes from factor analyses of the Affect Intensity Measure (Larsen, Diener, \& Emmons, 1986) and has been shown to correlate highly with other measures of negative affectivity (Rubin, Hoyle, \& Leary, 2012). Although the fourth measure, the Generalized Anxiety Disorder Scale (GADS; Spitzer, Kroenke, Williams, \& Lowe, 2006), was not designed as a general measure of negative affectivity, it includes items indicating negative affectivity and correlates highly with measures of negative affectivity (Hale, Klimstra, \& Meeus, 2010; Hettema, Neale, Myers, Prescott, \& Kendler, 2006). Like narrative centrality, there is substantial work showing a strong relation between negative affectivity and reactions to stressful events as measured by PTSD symptoms. In a review of 11 studies totaling 1,415 individuals, Rubin, Berntsen, and Bohni (2008) found an average correlation of measures of negative affectivity and PTSD symptoms of .43 . Both narrative affectivity and PTSD symptom severity involve a tendency to experience negative emotions, and part of their empirical relationship may be related to this conceptual overlap (Ormel, Rosmalen, \& Farmer, 2004). However, both concepts as measured here de- pend on many other properties, and so, it is unlikely that this one commonality is responsible for the relationships observed.

In terms of research strategy, we use a broad range of people and events to strengthen our claim that narrative centrality and negative affectivity play important roles in the severity of symptoms of posttraumatic stress. In order to obtain large samples for statistical purposes that vary along many dimensions, including a wide range of severity and kinds of negative events, we sampled college undergraduates and veterans not clinically assessed. However, we note that almost all the measures we use together here have also been used separately in clinical samples and tend to produce correlations with PTSD symptom severity similar to those for undergraduates (e.g., Brown et al., 2010; Davidson, Kudler, \& Smith, 1987; Hyer et al., 1994; Rubin et al., 2011). However, measures of narrative centrality tend to be quite high in PTSD samples (e.g., Rubin et al., 2011), and the accompanying reduction in variability reduces our ability to investigate interactions with measures of negative affectivity. We also attempt to vary the measures used for our key concepts across studies in order to ensure our findings are not specific to one measure but represent the underlying concepts of interest. This variety in participants, events, and measures makes it harder to make detailed comparisons across studies, but because the results are similar in our four studies, the generalizability of our conclusions is increased.

Study 1 builds on existing findings by using a large sample to examine the relationship of one measure of narrative centrality, the CES, and one measure of negative affectivity, N. Study 2 replicates Study 1 using different measures of negative affectivity and posttraumatic stress symptoms in a sample of veterans. Study 3 examines the broader concepts of narrative centrality and negative affectivity by including three measures of each. In all three studies, the measures of narrative centrality were made on the same event as the symptoms of stress, whereas the measures of negative affectivity were made without reference to this event. Study 4 explores the relation of narrative centrality and negative affectivity in a sample of measures that correlate with PTSD symptom severity. Consistent with our individual-differences approach, our primary analyses in all four studies are correlational including first-order correlations, multiple regression, structural equation modeling (SEM), and factor analysis. In our first three studies, we find an interaction between narrative centrality and negative affectivity such that high values of both measures were associated with levels of traumatic stress above what would be predicted by additive effects. As is discussed later, in the purely correlational approaches, the magnitude of this interaction is theoretically limited (McClelland \& Judd, 1993; Rogers, 2002; Whisman \& McClelland, 2005), and so, we also plot our data and examine slopes and differences in the means to interpret our results.

\section{Study 1: Symptoms of Posttraumatic Stress Predicted by One Measure of Narrative Centrality and Negative Affectivity}

The purpose of Study 1 is to obtain an initial estimate of the additive and interactive effects of narrative centrality and negative affectivity on symptoms of posttraumatic stress. Study 1 has a 
large sample size, but only two independent variables: $\mathrm{N}$ from the BFI (John et al., 1991) and the CES (Berntsen \& Rubin, 2006, 2007).

\section{Method}

Participants. We include data from 2,296 Duke University (Durham, NC) undergraduates (1,342 female; mean age of 19.05 years, $S D$ of 1.10) who completed our measures during a single session in one of six semesters between the fall semester of 2008 and the spring semester of 2012 .

Measures. The reliabilities of all measures are reported in Table 1.

Neuroticism (N). $\quad \mathrm{N}$ was assessed using the BFI (John et al., 1991), which is a 44-item measure of the broad personality domains of extraversion, neuroticism, agreeableness, conscientiousness, and openness. $\mathrm{N}$ is assessed by eight short phrases about behaviors such as becoming nervous easily or remaining calm in troubling situations (reverse scored).

Centrality of Event Scale (CES). The CES (Berntsen \& Rubin, 2006, 2007) measures the extent to which a traumatic memory forms a central component of personal identity, a turning point in the life story, and a reference point for everyday inferences. The short form of the CES, which we used, consists of seven items rated in relation to the most stressful or traumatic event in the person's life, which include "I feel that this event has become part of my identity," "This event has become a reference point for the way I understand myself and the world," "This event has permanently changed my life," "I often think about the effect this event have will have on my future," and "This event was a turning point in my life."

Posttraumatic Stress Disorder Checklist (PCL). In the PCL (Blanchard, Jones-Alexander, Buckley, \& Foneris, 1996; Weathers, Litz, Huska, \& Keane, 1994), participants nominate a specific stressful event and rate on 5-point scales from not at all to extremely how much they have been bothered by it on each of 17 symptoms of PTSD in the Diagnostic and Statistical Manual of Mental Disorders (4th ed., text rev., American Psychiatric Association, 2000).

Procedure. Our measures were part of a general Web-based screening of students in courses that require research participation in the Duke University Department of Psychology and Neuroscience, which occurs at the beginning of each academic semester. Our instruments were placed among those of other researchers from the department. Participants were asked to "please take a moment to think of what negative event or experience from your life is most troubling and stressful to you now" and then to complete the PCL and the seven-item CES with respect to that event.

\section{Results}

Table 1 presents the variables and their correlations. To examine whether there were interaction effects of our two independent variables, we created an interaction term, $\mathrm{N}^{*} \mathrm{CES}$, the product of the mean-centered $\mathrm{N}$ and CES. As can be seen in Table 1, there were significant correlations of $\mathrm{N}$ and the CES with the PCL. To ensure that $\mathrm{N}$ and CES have independent contributions, we performed simultaneous multiple regression analyses. Without the interaction term, the standardized beta weights, which were significant at the .0001 level, produced the equation PCL $=.29 \mathrm{~N}+$ $.45 \mathrm{CES}, R^{2}=.35$. The squared Type II semipartial correlations were $\mathrm{N}=.08$ and CES $=.19$. The common variance among these two variables was estimated to be .08 . Adding the product term produced a $\Delta R^{2}=.01, p<.0001$. Consistent with our claims, both $\mathrm{N}$ and the CES account for independent variance in symptoms of posttraumatic stress, and only about one quarter of the explanatory power was shared by narrative centrality, negative affectivity, and their interaction. In general, although the interaction term is useful in regression analyses for determining

Table 1

Means, Standard Deviations, Alphas, and Correlations of Variables in Studies 1 and 2

\begin{tabular}{|c|c|c|c|c|c|c|c|c|c|c|}
\hline \multirow[b]{2}{*}{ Variable } & \multicolumn{3}{|c|}{ Measure } & \multicolumn{7}{|c|}{ Correlations } \\
\hline & $M$ & $S D$ & $\alpha$ & 1 & 2 & 3 & 4 & 5 & 6 & 7 \\
\hline \multicolumn{11}{|l|}{ Study 1} \\
\hline 1. PCL & 30.07 & 11.34 & .91 & - & & & & & & \\
\hline 2. $\mathrm{N}$ & 2.86 & 0.74 & .84 & $.40^{*}$ & - & & & & & \\
\hline 3. CES & 2.55 & 1.14 & .93 & $.51^{*}$ & $.22^{*}$ & - & & & & \\
\hline \multicolumn{11}{|l|}{ Study 2} \\
\hline 1. IES-R & 29.93 & 22.67 & .96 & - & & & & & & \\
\hline 2. GADS & 8.55 & 6.36 & .93 & $.67^{*}$ & - & & & & & \\
\hline 3. CES & 3.28 & 1.29 & .95 & $.57^{*}$ & $.46^{*}$ & - & & & & \\
\hline \multicolumn{11}{|l|}{ Study 3} \\
\hline 1. PCL & 32.63 & 15.52 & .95 & - & & & & & & \\
\hline 2. $\mathrm{N}$ & 30.02 & 6.24 & .82 & $.29^{*}$ & - & & & & & \\
\hline 3. NA & 22.28 & 7.70 & .88 & $.39^{*}$ & $.52^{*}$ & - & & & & \\
\hline 4. AIM-NI & 27.84 & 7.21 & .81 & $.29^{*}$ & $.70^{*}$ & $.47^{*}$ & - & & & \\
\hline 5. CES & 2.81 & 1.09 & .97 & $.56^{*}$ & $.20^{*}$ & $.18^{*}$ & $.19^{*}$ & - & & \\
\hline 6. Closure & 30.95 & 13.27 & .88 & $-.44^{*}$ & $-.21^{*}$ & $-.20^{*}$ & $-.19^{*}$ & $-.46^{*}$ & - & \\
\hline 7. PTGI & 62.79 & 30.02 & .97 & $.38^{*}$ & -.03 & .04 & -.00 & $.59^{*}$ & $-.22^{*}$ & - \\
\hline
\end{tabular}

Note. Reliability is measured by Cronbach's alpha, $\alpha$. AIM-NI = Affect Intensity Measure-Negative Intensity; CES = Centrality of Event Scale; Closure = Closure Scale; GADS = Generalized Anxiety Disorder Scale; IES-R = Impact of Event Scale-Revised; $\mathrm{N}=\mathrm{Neuroticism}$; NA = Negative Affect; PCL $=$ Posttraumatic Stress Disorder Checklist; PTGI $=$ Posttraumatic Growth Inventory.

${ }^{*} p<.0001$. 
whether there is a significant contribution, its value in interpreting the size of that contribution is limited. For instance, Rogers (2002) calculated that with correlations of the magnitude similar to those we obtain using $\mathrm{N}$ and the CES to predict the PCL, the interaction term is limited theoretically to a maximum of roughly .06. Thus, we turn to the standard procedure of examining mean values and slopes.

The top panel of Figure 1 contains a descriptive graphic representation to help interpret the interaction of $\mathrm{N}$ and the CES. Given the luxury of 2,296 participants, we could form 15 groups determined by three levels of the CES and five of $\mathrm{N}$ and still produce smooth plots with small standard errors on each point. To produce nearly equal groups with simple-to-understand cutoffs, we divided the participants into the 811 who had a CES score less than 2.00, the 717 who had a CES between 2.00 and 3.00, and the 768 who had a CES greater than 3.00. The correlations between the PCL and $\mathrm{N}$ for the three CES groups were $.30, .35$, and .40 , respectively, indicating that the $\mathrm{N}^{*} \mathrm{CES}$ interaction can be seen as an increase in the relationship between $\mathrm{N}$ and PCL as the CES increased. To produce the plot in Figure 1, we then also grouped $\mathrm{N}$ into five bins of nearly equal numbers of participants: $\mathrm{N} \leq 2.20$, $n=444 ; 2.20<\mathrm{N} \leq 2.70, n=520 ; 2.70<\mathrm{N} \leq 3.10, n=431$; $3.10<\mathrm{N} \leq 3.55, n=484$; and $\mathrm{N}>3.55, n=417$. In terms of means, the group with the lowest CES and $\mathrm{N}$ values had a mean PCL of 21.38, near the minimum possible score of 17 , and the group with the highest CES and N values had a mean PCL of 43.97. Pencil-and-paper self-report measures cannot be used for diagnosis, but a commonly used self-report cutoff for the PCL that produces diagnostic accuracy for determining PTSD with a clinical diagnosis is 44 (Blanchard et al., 1996). The difference in means in the PCL for the high and low CES groups is 9.83 at the lowest level of $\mathrm{N}$ and 16.91 at the highest. Thus, CES, N, and their interaction account for substantial effects on the PCL.

\section{Discussion}

We investigated how the effects of narrative centrality and negative affectivity combine to account for symptoms of posttraumatic stress. As expected from earlier studies in which both measures had substantial weights in multiple regression models (Berntsen \& Rubin, 2007; Boelen, 2012; Rubin, Boals, \& Berntsen, 2008) and as confirmed here by the statistical analyses and by Figure 1, there was a significant interaction in the direction of a greater contribution of each measure as the other increased. The mean differences in the PCL with the CES, N, and their interaction, shown in Figure 1, were substantial. In addition, most of the variance that could be explained could be attributed to these two predictors rather than to variance shared among them.

\section{Study 2: Symptoms of Posttraumatic Stress Predicted by One Measure of Narrative Centrality and Negative Affectivity in a Sample of Military Veterans}

In Study 2, we tested a sample of military veterans. The majority nominated events related to combat and other military traumas. In addition, we used a different measure of PTSD symptoms and negative affectivity to demonstrate that the results obtained in Study 1 were not specific to the measures used in that study.
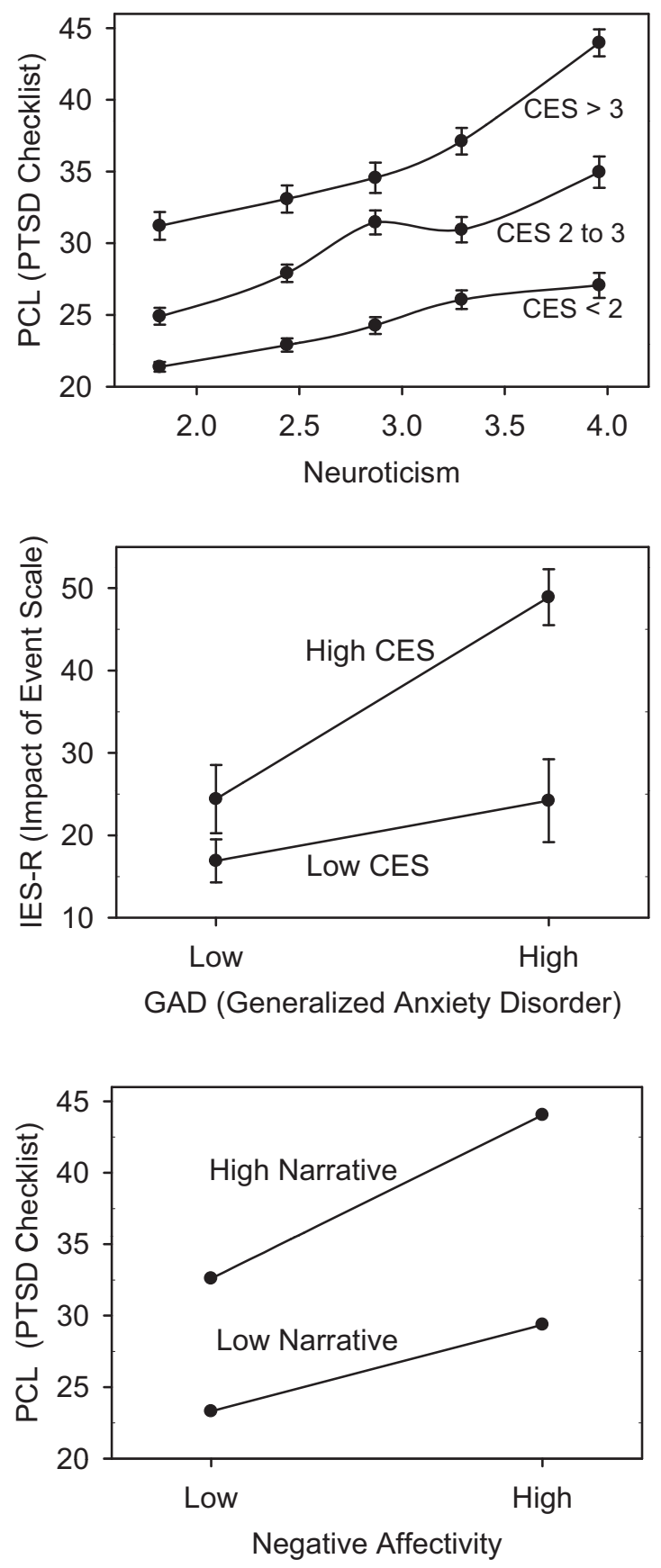

Figure 1. The top panel plots posttraumatic symptom severity from Study 1 as measured by the PCL as a function of Neuroticism for three levels of the Centrality of Event Scale (CES). Error bars are standard errors. The middle panel plots posttraumatic symptom severity from Study 2 as measured by the IES-R as a function of the GADS and CES. Error bars are standard errors. The bottom panel provides an analogous plot for Study 3 , plotting posttraumatic symptom severity as a function of the derived composite measure of negative affectivity for levels of narrative centrality one standard deviation above and below the mean.

\section{Method}

Participants and procedure. We include data from 104 military veterans recruited from university campuses (22 female; 
mean age of 31.69 years, $S D$ of 7.42$)$. A total of $79(73 \%)$ indicated they had been deployed to a war zone. The majority $(n=$ 90) were students at the University of North Texas (Denton, TX). The measures were completed as part of an online survey that included several instruments. Participants were asked to complete the Impact of Event Scale-Revised (IES-R) in reference to "stressful military experiences" and then complete the CES in reference to the same event.

Measures (not described in Study 1). The reliabilities of all measures are reported in Table 1.

Generalized Anxiety Disorder Scale (GADS). The GADS is a seven-item measure designed to assess generalized anxiety disorder (Spitzer et al., 2006). Items include statements such as feeling nervous or anxious and having trouble relaxing which are rated from 0 (not at all) to 3 (nearly every day).

Impact of Event Scale-Revised (IES-R). The IES-R is a 22item questionnaire designed to measure PTSD symptoms (Weiss \& Marmar, 1997). The IES-R consists of three subscales that measure the B (intrusiveness), C (avoidance), and D (hyperarousal) symptoms for PTSD. Items were scored on a 5-point scale from 0 (not at all) to 4 (extremely).

\section{Results}

Table 1 presents the variables and their correlations. To examine whether there were multiplicative effects of our two independent variables, we created an interaction term, GADS*CES, the product of the mean-centered GADS and CES. As can be seen in Table 1, there were significant correlations of the GADS and the CES with the IES-R. To ensure that GADS and CES have independent contributions, we performed simultaneous multiple regression analyses. Without the interaction term, the standardized beta weights, which were significant at the .0001 level, produced the equation IES-R $=.52 \mathrm{GADS}+.33 \mathrm{CES}, R^{2}=.52$. The squared Type II semipartial correlations were GADS $=.31$ and CES $=.16$. The common variance among these two variables was estimated to be .05 . Adding the product term produced a $\Delta R^{2}=.02, p<.05$.

The middle panel of Figure 1 contains a descriptive graphic representation to help interpret the interaction of the GADS and the CES. Because we had many fewer participants than in Study 1, we did median splits for the figure. The main effects and interactions are clearly shown and are similar to those of Study 1, which are in the top panel. In terms of means, the group with the lowest CES and GADS values had a mean IES-R of 14.84 (the minimum possible score is 0), and the group with the highest CES and GADS values had a mean IES-R of 48.41. A commonly used self-report cutoff for the IES-R that produces diagnostic accuracy for determining PTSD with a clinical diagnosis is 33 (Creamer, Bell, \& Faillia, 2003). The difference in mean in the IES-R for the high and low CES groups is 9.64 at the lowest level of GADS and 25.14 at the highest. Thus, CES, GADS, and their interaction account for substantial effects on the IES-R.

\section{Discussion}

The results replicated those obtained in Study 1, but this time with a sample of veterans and different measures of negative affectivity (GADS in lieu of N) and PTSD symptoms (IES-R in lieu of the PCL).

\section{Study 3: Symptoms of Posttraumatic Stress Predicted by Three Measures of Narrative Centrality and Negative Affectivity}

In Study 3, we use three quantitative individual-differences measures of narrative centrality and three of negative affectivity to ensure that our theoretical claims extend beyond the single particular measures that we used in Studies 1 and 2. To test the hypothesis that we have one underlying dimension of narrative centrality and one of negative affectivity, we first report the correlations within and between our three measures of each dimension and then use SEM to formally analyze the relation of the two latent variables corresponding to the dimensions.

\section{Method}

Participants and procedure. We include data from 488 University of North Texas undergraduates (337 female; mean age of 20.73 years, $S D$ of 3.28) who completed our measures in the 2011-2012 academic year. The measures were completed as part of an online survey that included several instruments. Participants identified their most traumatic event by completing the Stressful Life Events Screen Questionnaire-Revised (SLESQ-R; Goodman, Corcoran, Turner, Yuan, \& Green, 1998). For the purposes of another study being conducted with this data set, if participants indicated a traumatic event on the SLESQ-R that was of a sexual nature (e.g., sexual assault), they were asked to select this event as their most traumatic. Thus, the current data set oversampled sexrelated events. Participants then completed the PCL, CES, Closure Scale, and PTGI in reference to their most traumatic event.

Measures (not described in Studies 1 or 2). The reliabilities of all measures are reported in Table 1.

Affect Intensity Measure-Negative Intensity (AIM-NI). The AIM-NI comes from factor analysis of the AIM (Larsen et al., 1986). It includes eight items that reflect a global tendency to react intensely versus calmly, such as friends considering the participant as a tense person or the participant's negative moods being mild (reverse scored). The AIM-NI has good internal consistency and has been shown to be highly correlated to other measures of negative affectivity (Rubin et al., 2012).

Centrality of Event Scale (CES). Unlike Study 1, here we use the full 20-item scale.

Closure Scale. The Closure Scale (Beike \& Wirth-Beaumont, 2005) measures the extent to which a negative event is no longer of current concern. The scale asks participants to rate their agreement to five statements, including "I have complete closure on this event" and "The event seems like ancient history." Participants rate each item on a scale of 1 to 10 , and the sum score is reported.

Negative Affect (NA). NA comes from the PANAS (Watson et al., 1988), which includes two 10-item subscales that measure the tendency to experience positive and negative affect. For the NA, participants rate their tendency to experience 10 negative feelings.

Posttraumatic Growth Inventory (PTGI). The PTGI (Tedeschi \& Calhoun, 1996) is a 21-item questionnaire designed to measure the results of a crisis occurring in an individual's life such as, because of the crisis, the participant knows that difficulties can be better handled. Items are appraised on a scale from 0 (I did not 
experience this change as a result of my crisis) to 5 (I experienced this change to a very great degree as a result of my crisis).

\section{Results}

As shown in Table 1, correlations between the Closure Scale and the other measures are generally negative because events that are of less current concern are associated with lower PTSD symptoms severity, CES, and PTGI measures. The absolute values of the correlations of all the independent variables with the PCL are between .29 and .56. The absolute values of the correlations between measures of narrative centrality and measures of negative affectivity are between .00 and .21 . The absolute values of the correlations of the three measures of negative affectivity are between .47 and .70 , and the absolute values of the correlations of the three measures of narrative centrality are between .46 and .59 , with the exception of the correlation between the PTGI and the Closure Scale, which has an absolute value of .22. However, the maximum absolute value of the correlation between the PTGI and any of the three negative affectivity measures is .04. Thus, we have general descriptive support for our claim of two different kinds of measures leading to symptoms of posttraumatic stress.

We used SEM to verify that the correlations between the measures of negative affectivity and narrative centrality could be attributed to two underlying dimensions. Maximum-likelihood estimation of a model with two correlated factors revealed excellent fit to the data, $\chi^{2}(8, N=488)=32.44$, comparative fit index $=$ .97 , root-mean-square error of approximation $=.07$. Standardized parameter estimates are presented in Figure 2. All loadings were significant and indicative of substantial commonality among the indicators of each factor. The factors were modestly correlated with each other. Model comparisons indicated that this model provides a better account of the data than a one-factor model, $\Delta \chi^{2}(1)=292.06, p<.0001$, or a two-factor model in which the factors are uncorrelated, $\Delta \chi^{2}(1)=24.00, p<.0001$.

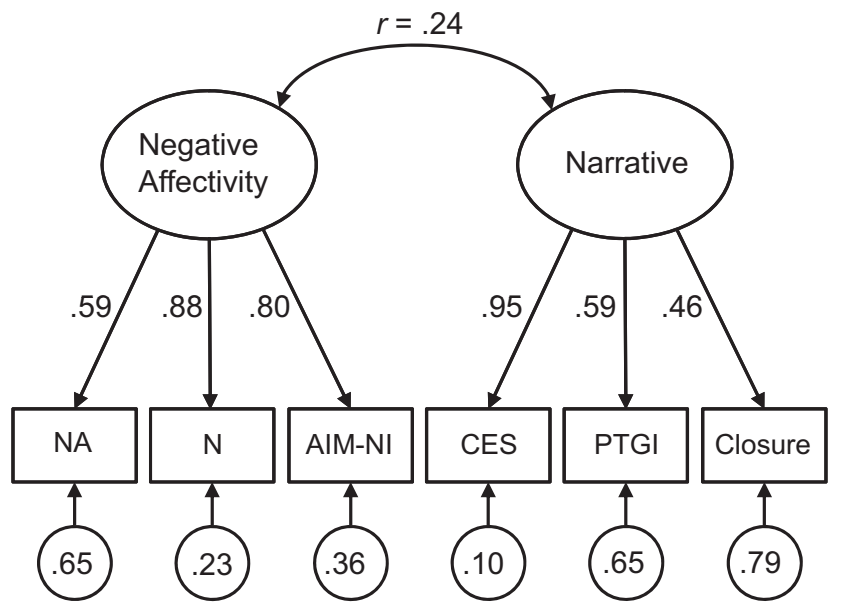

Figure 2. A structural equation model demonstrating that the correlations between the measures of narrative centrality and negative affectivity can be attributed to two underlying dimensions. AIM-NI = Affect Intensity Measure-Negative Intensity; CES = Centrality of Event Scale; Closure = Closure Scale; $\mathrm{N}=$ Neuroticism; NA = Negative Affect; PTGI = Posttraumatic Growth Inventory.
We used two methods to examine the main effects and interaction of these latent variables as predictors of PCL scores. Staying within the SEM context, we regressed PCL scores on the two latent variables and a latent interaction term. The coefficients for all effects were significant (minimum $p<.005$ ). Follow-up analyses for latent interaction tests have not yet been developed, so we shifted to a multiple regression context to evaluate the pattern underlying the significant interaction effect. We output factor scores for the two latent variables, then created a product term corresponding to the interaction effect. The regression predicting PCL from these terms was significant, $F(3,484)=76.37, p<$ $.001, R^{2}=.32$. Follow-up simple slopes analyses were used to probe the significant interaction effect. As shown in the bottom panel of Figure 1, at both one standard deviation above and below the mean on narrative centrality, the relation between negative affectivity and PCL scores was positive. In both cases, the slopes were significant, the interaction effect reflecting a steeper slope at high negative affectivity $(b=.36, t=6.76, p<.001)$ than at low negative affectivity $(b=.17, t=3.01, p<.01)$. Using the simple slopes from Figure 1 at the lowest narrative centrality and negative affectivity values the PCL was 23.31, near the minimum possible score of 17 , and at the highest narrative centrality and negative affectivity values, the PCL was 44.05, near a commonly used self-report cutoff of 44 for diagnostic accuracy compared with a clinical diagnosis of PTSD (Blanchard et al., 1996). The difference in means in the PCL for the high and low narrative centrality values is 9.29 at the lowest level of negative affectivity and 14.68 at the highest. Thus, narrative centrality, negative affectivity, and their interaction account for substantial effects on the PCL.

\section{Discussion}

Study 3 demonstrated that latent variables underlying three measures of negative affectivity and three measures of narrative centrality acted in much the same way as our single measures used in Studies 1 and 2.

\section{Study 4: An Examination of Other Correlates of Symptoms of Posttraumatic Stress}

Here, we investigate whether individual-differences measures that correlate with symptoms of posttraumatic stress in general tend to divide into those that correlate with narrative centrality and those that correlate with negative affectivity. We report on measures that were sampled widely and for purposes unrelated to the current article that correlated .35 or greater with the PCL. Thus, we have an exploratory study using a convenience sample of measures, though it is not clear how one would sample measures that correlate with PTSD symptoms in a more theoretically motivated way.

\section{Method}

Participants and procedure. We include data from 987 University of North Texas undergraduates (667 female; mean age of 19.78 years, $S D$ of 2.20) who completed the measures in the fall semester of 2008. Their data were not suitable for the analysis done in Study 3 because they included only one measure of negative affectivity. As in Studies 1 and 3, the measures were 
completed as part of online surveys that included instruments from multiple researchers.

Measures (not described in Studies 1 or 2). We report on all measures available to us that correlated .35 or greater with the PCL. The reliabilities are reported in Table 2.

Avoidance Coping. Avoidance Coping is one of three combinations of subscales from the Brief COPE (Carver, 1997). It measures avoidant coping style; the other two measures are problem-focused and emotion-focused coping. The Brief COPE itself is a 28-item self-report questionnaire designed to measure a broad range of coping styles. Participants rated the extent to which they engage in various coping behaviors on a scale from 1 ( $I$ haven't been doing this at all) to 4 (I've been doing this a lot).

Experiential Avoidance. Experiential Avoidance comes from the Acceptance and Action Questionnaire (Bond et al., 2011), a 10-item self-report measure of psychological flexibility, which refers to the experiential willingness to make contact with the present moment and persist or change behavior in accordance with one's values. It actually measures the inverse of avoiding experiences. Example items ask about things such as whether it is alright to remember something unpleasant.

Insomnia Severity Index (ISI). The ISI (Bastien, Vallieres, \& Morin, 2001) is a seven-item self-report measure designed to assess perceived severity of insomnia. Items include questions about things such as how noticeable to others one's sleeping problems are. Each item uses a scale from 1 (not at all satisfied) to 5 (very much satisfied). Items are summed, yielding a minimum score of 7 and a maximum score of 35 .

Quick Inventory of Depressive Symptomatology-Self-Report (QIDS-SR). The QIDS-SR (Rush et al., 2003) measures depression. The 16-item questionnaire asks individuals to rate how much each depression symptom has been applicable within the last 7 days. Symptoms include items about sleep patterns, mood, weight change, and loss of general interest.

White Bear Suppression Inventory. The White Bear Suppression Inventory is a 15-item instrument rated on a 5-point scale from 1 (strongly disagree) to 5 (strongly agree) that measures the general tendency to suppress unwanted negative thoughts (Wegner \& Zanakos, 1994).

\section{Results and Discussion}

By way of a partial replication of Table 1, we note that in the University of North Texas data, which are independent of the Study 3 data, the same three measures of narrative centrality correlated to about the same extent. The CES correlated with the Closure Scale -.57 and with the PTGI .60. The Closure Scale and the PTGI correlated -.32 (all $p$ s $<.0001$ ). Thus, as in Study 3 , the CES correlates more highly with the Closure Scale and the PTGI than the latter measures correlate with each other. In contrast, the only measure of negative affectivity we had, NA, again had similar lower correlations with the three narrative centrality variables. The NA correlated with the CES .15 , the Closure Scale -.15 , and the PTGI .08 (all $p$ s $<.01$ ).

As shown in Table 2, the measures included in this study tend to correlate highly with either measures of negative affectivity or narrative centrality, but not both. Moreover, they correlate at least as highly with either NA or the CES as with the PCL, with which they had to correlate at least .35 to be included in the analysis.

As shown in Table 2, to further investigate this result, we performed a principal axes extraction followed by oblique rotation exploratory factor analyses for all variables except the PCL. The first four eigenvalues were $3.19,1.15,0.13$, and 0.05 . A popular method of determining the correct number of components or factors in an exploratory factor analysis is to count and interpret the number of factors that have an eigenvalue greater than 1.00. A second informal criterion is to look for a sharp break in the magnitude of the rank-ordered eigenvalues (i.e., in a scree plot) in order to determine the number of factors after which including additional factors adds no explanatory power to the solution. A more formal way to determine the number of factors is the use of parallel analysis, in which the number of factors/components to retain is determined by comparing the eigenvalues for different numbers of factors/components with those of simulated solutions based on random variables as a means of ruling out eigenvalues that do not differ from what would have been expected by chance (Hoyle \& Duvall, 2004). All three criteria pointed to the retention of two factors. The rightmost columns of Table 2 contain the factor patterns and commonalities, which showed the expected two-

Table 2

Means, Standard Deviations, Alphas, Correlations With the PCL, NA, and CES, and Factor Analysis of Measures From Study 4

\begin{tabular}{|c|c|c|c|c|c|c|c|c|c|}
\hline \multirow[b]{2}{*}{ Measure } & \multirow[b]{2}{*}{$M$} & \multirow[b]{2}{*}{$S D$} & \multirow[b]{2}{*}{$\alpha$} & \multicolumn{3}{|c|}{ Correlation with } & \multicolumn{3}{|c|}{ Factor analysis } \\
\hline & & & & PCL & N/NA & CES & 1 & 2 & $h^{2}$ \\
\hline PCL & 27.78 & 12.80 & .94 & - & .35 & .53 & & & \\
\hline NA & 21.79 & 6.93 & .86 & .35 & - & .15 & .71 & -.06 & .48 \\
\hline Avoidance Coping & 17.39 & 4.89 & .66 & .42 & .56 & .19 & .72 & .02 & .51 \\
\hline QIDS-SR & 6.55 & 4.16 & .76 & .43 & .52 & .22 & .75 & .00 & .57 \\
\hline Experiential Avoidance & 4.98 & 1.04 & .86 & -.47 & -.50 & -.30 & -.70 & -.10 & .55 \\
\hline White Bear & 49.95 & 10.93 & .90 & .35 & .44 & .19 & .61 & .03 & .38 \\
\hline ISI & 9.06 & 5.52 & .85 & .38 & .43 & .18 & .63 & -.01 & .39 \\
\hline CES & 2.43 & 1.23 & .94 & .53 & .15 & - & .02 & .80 & .66 \\
\hline PTGI & 30.96 & 28.28 & .97 & .41 & .08 & .60 & -.10 & .67 & .42 \\
\hline Closure & 31.87 & 13.47 & .89 & -.52 & -.13 & -.57 & -.11 & -.58 & .39 \\
\hline
\end{tabular}

Note. Reliability is measured by Cronbach's alpha, $\alpha$. For all correlations, $p<.0001 . \mathrm{CES}=$ Centrality of Event Scale; Closure $=$ Closure Scale; ISI $=$ Insomnia Severity Index; N = Neuroticism; NA = Negative Affect; PCL = Posttraumatic Stress Disorder Checklist; PTGI = Posttraumatic Growth Inventory; QIDS-SR = Quick Inventory of Depressive Symptomatology-Self-Report; White Bear = White Bear Suppression Inventory. 
factor solution, with the two factors correlating .34. Thus, for a convenience sample of individual-differences measures, our distinction between measures of narrative centrality and measures of negative affectivity is supported. Note that we are claiming that our measures form two factors: one related to negative affectivity and the other to narrative centrality. We are not claiming that the measures correlate with the PCL only because they correlate with the CES or NA; in multiple regression models, the measures listed in Table 1 contribute independent variance to the prediction of the PCL beyond that of both the CES and NA (all $p s<.05$ ).

Our measures and perhaps others correlate with the PCL principally because they are related to either narrative centrality or negative affectivity. It is not clear what a more systematic sampling of measures that correlate with symptoms of posttraumatic stress would be, but future work looking for measures that would be strong exceptions to our claims would help guide the search for predictors of symptoms of posttraumatic stress. Possible exceptions could be measures of social support and attachment, which should have lower correlations with narrative centrality and negative affect.

\section{General Discussion}

We have argued that there are two conceptually distinct classes of measures that are major contributors to symptoms of posttraumatic stress. One class of measures includes characteristics of the person as measured by the general concept of negative affectivity. We used four measures of negative affectivity that differed in form and conceptual motivation. $\mathrm{N}$ is a direct attempt at measuring neuroticism in the context of four other personality traits. AIM-NI is one factor from a scale developed to measure affect intensity and as such focuses on emotional reactions. NA from the PANAS is based on a conception of two factors underlying emotions related to psychopathology rather than on a set of factors that are intended to describe all important aspects of personality. GADS is a measure used to assess generalized anxiety disorder. When we examined a sample of measures that correlated with symptoms of posttraumatic stress, except for our three narrative centrality measures, all measures correlated highly with measures of negative affectivity. Moreover, the measures from this sample had a broad set of theoretical motivations, including the intrusive memories and thoughts of the White Bear Suppression Inventory, avoidancerelated measures such as Avoidance Coping and Experiential Avoidance, and measures of depression and insomnia. Thus, although we are using a single concept of negative affectivity, the set of behaviors and emotions judged in our various measures is quite broad. One property they share in common is that they are about the way individuals react to a wide range of events, not a single event.

The other class included measures of the centrality of the individual's narrative of a single negative event to his or her life story or narrative identity rather than fairly stable characteristics of the individual. We could find only three measures of narrative centrality. The CES measures how important a particular event is to a person's identity, life story, and way of judging future events. The Closure Scale measures how much a once-important negative event is now resolved and no longer affects current goals and judgments. Thus, the CES and the Closure Scale can be seen as complementary measures of similar concepts; if a negative event is central to narrative identity and life story, then issues related to it tend not to be resolved. The PTGI has a completely different motivation and assesses the extent to which one believes one has grown from the negative event; even though the event is seen as having positive consequences, the PTGI still measures how central the negative event is to narrative identity and life story. Because the PTGI correlates positively with the CES and the PCL, we know that what matters is that individuals' narratives of their negative events include the perception that the events are central to their life story, not whether the events are seen as having negative consequences. Other narrative centrality measures of negative events that correlate with symptoms of posttraumatic stress may exist, but we were not aware of them. Absent are measures of coherence and fragmentation, both because they are about way the narratives are constructed rather than addressing the meaning of the memory as do the ratings of narrative centrality and also because they do not provide consistent correlations with symptoms of posttraumatic stress (for reviews, see Berntsen \& Rubin, 2013; Rubin, 2011; Rubin, Boals, \& Berntsen, 2008).

We found clear empirical support for the conceptual dichotomy of measures of the person and measures of the person's evaluation of a single negative event as currently central to his or her narrative identity or life story in three studies and in our exploration of other correlates of symptoms of posttraumatic stress. Both factors contributed independently in multiple regressions predicting symptoms of posttraumatic stress, and there was an interaction in the direction of each factor increasing for higher levels of the other. Moreover, the independent contributions are substantial, leaving little common predictive variance. Nonetheless, there was a small but consistent association between the measures of narrative centrality and negative affectivity. One possibility is that increased negative affectivity contributes to a tendency to create narratives about negative experiences that make them more central to identity, though this remains speculation.

Examining means instead of variance, the overall combined quantitative effects of narrative centrality and negative affectivity are substantial; the measure of PTSD symptom severity at the lowest value in all three panels of Figure 1 is near the lowest score possible on the symptom severity tests. In contrast, mean symptom severity at the highest value in all three panels is at or above the paper-and-pencil self-report cutoff for diagnostic accuracy for determining PTSD with a clinical diagnosis (Blanchard et al., 1996; Creamer et al., 2003). Although the interactions accounted for little added variance, as is usually the case in noncrossover interactions (McClelland \& Judd, 1993; Rogers, 2002; Whisman \& McClelland, 2005), the interactions were statistically reliable and substantial in terms of mean differences. For the first three studies, as shown in Figure 1, over a variety of measures and statistical techniques, the differences between high and low narrative centrality were about twice as large at the high extreme of negative affectivity compared to the low extreme.

\section{Narrative Centrality as a Component of Personality}

Including measures of both negative affectivity and narrative centrality allows us to explore a conceptual integration of approaches of personality relevant to stress reactions. McAdams (1996) proposed three conceptual levels of measurement of personality. Level 1 includes broad, decontextualized, dispositional 
traits such as negative affectivity. Level 2 includes personal strivings, life tasks, defense mechanisms, coping strategies, and other motivational, developmental, and strategic constructs, which are contextualized in time, place, or role. Level 3 includes narrative identity in the form of a life story narrative that

manifests a particular (a) structure and content, (b) function, and (c) developmental course, and it may be further described in terms of (d) individual differences in types of self stories and (e) the extent to which a life story facilitates adaptation or reinforces pathology for the person whose identity the story is. (McAdams, 1996, pp. 301-302)

Integrating over these three levels as fully as McAdams proposed is not possible using quantitative scales of narrative that can be directly compared to his Level 1 dispositional scales. However, we approach this integration using scales of negative affectivity that are clearly in Level 1 of McAdams's model and scales of narrative centrality that quantify key aspects of Levels 2 and 3 of his model.

Moreover, although each of our measures of narrative centrality refers to a single event, there is a traitlike quality to the ratings that extends across events in that people with PTSD tend to rate all events as higher in the CES and PTGI (Boals \& Schuettler, 2011; Rubin, Boals, \& Berntsen, 2008; Schuettler \& Boals, 2011). This traitlike quality over events may relate to the stable individualdifferences attribute of self-focused attention, which reflects a heightened awareness of self-referent, internally generated information (Ingram, 1990). In addition, private self-awareness, in which the focus is from a personal perspective, and public selfawareness, in which the focus is from the perspective of others, may each contribute to psychological disorders including depression and PTSD, as might ruminative self-focus, as opposed to a more productive reflective self-focus (Fejfar \& Hoyle, 2000; Mor \& Winquist, 2002; Teasdale \& Green, 2004). Moreover, ruminative self-focus correlates with negative affectivity, and so, selffocused attention could provide at least a partial account of the correlations we observe between narrative centrality and negative affectivity (Teasdale \& Green, 2004).

\section{Limitations}

A broad theoretical dichotomy, such as the one made here, will certainly have limitations and flaws as it is developed. One major limitation is that in our studies, causal claims cannot be made because measurement occurs at a single time after the trauma. Thus, we use prediction only in the statistical sense employed in multiple regression models in which variance in one variable is accounted for by shared variance in other variables. This is not different from most other studies of the effects of traumatic events. Even psychosocial factors labeled as risk factors in the literature are usually measured retrospectively and therefore are influenced by everything that occurred up to a time of measurement, which is most often after the negative event. Monitoring measures of narrative centrality and negative affectivity over time and especially during therapy could elucidate potential mechanisms of change. A second limitation is that we use nonclinical samples, thus demonstrating the value of our theoretical claim and its potential application in general populations but not indicating the magnitude of any effects in the range of traumas and symptoms present in clinical populations. However, as reviewed in the introduction, based on the existing data on measures of narrative centrality and negative affectivity in clinical populations, there is good reason to expect that the independent contribution of narrative centrality and negative affectivity would hold. Nonetheless, replications in clinical samples and in studies in which the course of changes in symptoms, narrative centrality, and negative affectivity are measured longitudinally are needed to clarify the processes involved.

\section{Practical Implications}

The distinction between narrative centrality and negative affectivity can be seen in existing therapies for PTSD. Most therapies attempt to change the narrative evaluations of negative events rather than the memory of the event itself or how central it is to the life story or narrative identity (Bryant, 2011; Hembree \& Foa, 2010). Our assumption is that reducing the negative evaluation of the memory provides closure and makes the memory less central and in this way reduces the frequency with which the memory is recalled. The frequent recall of a negative event, rather than the valence of what one attempts to make of the event's sequelae, may be what matters and may help explain why the PTGI correlates positively with the PCL (Boals \& Schuettler, 2011).

The distinction between measures relating to the person and measures relating to narratives about an event appears to be an extremely useful one for approaching the understanding of responses to stressful events, the clearest being PTSD. However, the dichotomy may be useful more broadly if one views disorders, such as depression, as related to specific events or the accumulation of specific events. Moreover, although we have not demonstrated effects in clinical samples, we have demonstrated effects that are large enough to be of practical importance in nonclinical populations.

\section{References}

American Psychiatric Association. (2000). Diagnostic and statistical manual of mental disorders (4th ed., text rev.). Washington, DC: Author.

American Psychiatric Association. (2013). Diagnostic and statistical manual of mental disorders (5th ed.). Washington, DC: Author.

Bastien, C. H., Vallieres, A., \& Morin, C. M. (2001). Validation of the Insomnia Severity Index as an outcome measure for insomnia research. Sleep Medicine, 2, 297-307. doi:10.1016/S1389-9457(00)00065-4

Baumeister, R. F., \& Newman, L. S. (1994). How stories make sense of personal experiences: Motives that shape autobiographical narratives. Personality and Social Psychology Bulletin, 20, 676-690. doi:10.1177/ 0146167294206006

Beike, D. R., Adams, L. P., \& Naufel, K. Z. (2010). Closure of autobiographical memories moderates their directive effect on behavior. Memory, 18, 40-48. doi:10.1080/09658210903405729

Beike, D. R., \& Wirth-Beaumont, E. T. (2005). Psychological closure as a memory phenomenon. Memory, 13, 574-593. doi:10.1080/ 09658210444000241

Berntsen, D., \& Rubin, D. C. (2006). The Centrality of Event Scale: A measure of integrating a trauma into one's identity and its relation to post-traumatic stress disorder symptoms. Behaviour Research and Therapy, 44, 219-231. doi:10.1016/j.brat.2005.01.009

Berntsen, D., \& Rubin, D. C. (2007). When a trauma becomes a key to identity: Enhanced integration of trauma memories predicts posttraumatic stress disorder symptoms. Applied Cognitive Psychology, 21, 417-431. doi:10.1002/acp.1290

Berntsen, D., \& Rubin, D. C. (2013). Involuntary memories and dissociative amnesia: Assessing key assumptions in PTSD research. Clinical 
Psychological Science. Advance online publication. doi:10.1177/ 2167702613496241

Blanchard, E. B., Jones-Alexander, J., Buckley, T. C., \& Foneris, C. A. (1996). Psychometric properties of the PTSD Checklist (PCL). Behaviour Research and Therapy, 34, 669-673. doi:10.1016/00057967(96)00033-2

Boals, A. (2010). Autobiographical memories that have become central to identity: Gender differences in the Centrality of Events Scale for positive and negative events. Applied Cognitive Psychology, 24, 107-121. doi: 10.1002/acp. 1548

Boals, A., Banks, J. B., Hathaway, L. M., \& Schuettler, D. (2011). Coping with stressful events: Use of cognitive words in stressful narratives and the meaning-making process. Journal of Social and Clinical Psychology, 30, 378-403. doi:10.1521/jscp.2011.30.4.378

Boals, A., \& Schuettler, D. (2011). A double-edged sword: Event centrality, PTSD and posttraumatic growth. Applied Cognitive Psychology, 25, 817-822. doi:10.1002/acp.1753

Boelen, P. A. (2009). The centrality of a loss and its role in emotional problems among bereaved people. Behaviour Research and Therapy, 47, 616-622. doi:10.1016/j.brat.2009.03.009

Boelen, P. A. (2012). A prospective examination of the association between the centrality of a loss and post-loss psychopathology. Journal of Affective Disorders, 137, 117-124. doi:10.1016/j.jad.2011.12.004

Bond, F. W., Hayes, S. C., Baer, R. A., Carpenter, K. M., Guenole, N., Orcutt, H. K., . . . Zettle, R. D. (2011). Preliminary psychometric properties of the Acceptance and Action Questionnaire-II: A revised measure of psychological flexibility and experiential avoidance. Behavior Therapy, 42, 676-688. doi:10.1016/j.beth.2011.03.007

Brewin, C. R., Andrews, B., \& Valentine, J. D. (2000). Meta-analysis of risk factors for posttraumatic stress disorder in trauma-exposed adults. Journal of Consulting and Clinical Psychology, 68, 748-766. doi: 10.1037/0022-006X.68.5.748

Brown, A. D., Antonius, D., Kramer, M., Root, J. C., \& Hirst, W. (2010). Trauma centrality and PTSD in veterans returning from Iraq and Afghanistan. Journal of Traumatic Stress, 23, 496-499. doi:10.1002/jts .20547

Bruner, J. S. (1986). Actual minds, possible worlds. Cambridge, MA: Harvard University Press.

Bryant, R. (2011). Psychological interventions for trauma exposure and PTSD. In D. J. Stein, M. J. Friedman, \& C. Blanco (Eds.), Posttraumatic stress disorder (pp. 171-202). Hoboken, NJ: WileyBlackwell. doi:10.1002/9781119998471.ch5

Carver, C. S. (1997). You want to measure coping but your protocol's too long: Consider the Brief COPE. International Journal of Behavioral Medicine, 4, 92-100. doi:10.1207/s15327558ijbm0401_6

Caspi, A., Roberts, B. W., \& Shiner, R. L. (2005). Personality development: Stability and change. Annual Review of Psychology, 56, 453-484. doi:10.1146/annurev.psych.55.090902.141913

Costa, P. T., Herbst, J. H., McCrae, R. R., \& Siegler, I. C. (2000). Personality at midlife: Stability, intrinsic maturation, and response to life events. Assessment, 7, 365-378. doi:10.1177/107319110000700405

Coyne, J. C., \& Tennen, H. (2010). Positive psychology in cancer care: Bad science, exaggerated claims, and unproven medicine. Annals of Behavioral Medicine, 39, 16-26. doi:10.1007/s12160-009-9154-z

Crawley, R. A. (2010). Closure of autobiographical memories: The effects of written recounting from first- or third-person visual perspective. Memory, 18, 900-917. doi:10.1080/09658211.2010.524650

Creamer, M., Bell, R., \& Failla, S. (2003). Psychometric properties of the Impact of Event Scale-Revised. Behaviour Research and Therapy, 41, 1489-1496. doi:10.1016/j.brat.2003.07.010

Davidson, J., Kudler, H., \& Smith, R. (1987). Personality in chronic post-traumatic stress disorder: A study of the Eysenck inventory. Journal of Anxiety Disorders, 1, 295-300. doi:10.1016/08876185(87)90009-0
Digman, J. M. (1990). Personality structure: Emergence of the five-factor model. Annual Review of Psychology, 41, 417-440. doi:10.1146/ annurev.ps.41.020190.002221

Fejfar, M. C., \& Hoyle, R. H. (2000). Effect of private self-awareness on negative affect and self-referent attribution: A quantitative review. Personality and Social Psychology Review, 4, 132-142. doi:10.1207/ S15327957PSPR0402_02

Fivush, R., \& Hayden, C. A. (Eds.). (2003). Autobiographical memory and the construction of a narrative self: Developmental and cultural perspectives. Mahwah, NJ: Erlbaum.

Frazier, P., Tennen, H., Gavian, M., Park, C., Tomich, C., \& Tashiro, T. (2009). Does self-reported posttraumatic growth reflect genuine positive change? Psychological Science, 20, 912-919. doi:10.1111/j.1467-9280 2009.02381.x

Freeman, M. (1993). Rewriting the self. New York, NY: Routledge.

Goodman, L. A., Corcoran, C., Turner, K., Yuan, N., \& Green, B. L. (1998). Assessing traumatic event exposure: General issues and preliminary findings for the Stressful Life Events Screening Questionnaire. Journal of Traumatic Stress, 11, 521-542. doi:10.1023/A: 1024456713321

Hale, W. W., Klimstra, T. A., \& Meeus, W. H. J. (2010). Is the generalized anxiety disorder symptom of worry just another form of neuroticism? A 5 -year longitudinal study of adolescents from the general population. Journal of Clinical Psychiatry, 71, 942-948. doi:10.4088/JCP $.09 \mathrm{~m} 05506 \mathrm{blu}$

Hembree, E. A., \& Foa, E. B. (2010). Cognitive behavioral therapies for PTSD. In G. M. Rosen \& C. B. Frueh (Eds.), Clinician's guide to posttraumatic stress disorder (pp. 177-203). Hoboken, NJ: Wiley.

Hettema, J. M., Neale, M. C., Myers, J. M., Prescott, C. A., \& Kendler, K. S. (2006). A population-based twin study of the relationship between neuroticism and internalizing disorders. American Journal of Psychiatry, 163, 857-864. doi:10.1176/appi.ajp.163.5.857

Hoyle, R. H., \& Duvall, J. L. (2004). Determining the number of factors in exploratory and confirmatory factor analysis. In D. Kaplan (Ed.), Handbook of quantitative methodology for the social sciences (pp. 301-315). Thousand Oaks, CA: Sage. doi:10.4135/9781412986311.n16

Hyer, L., Braswell, L., Albrecht, B., Boyd, S., Boudewyns, P., \& Talbert, S. (1994). Relationship of NEO-PI to personality styles and severity of trauma in chronic PTSD victims. Journal of Clinical Psychology, 50, 699-707. doi:10.1002/1097-4679(199409)50:5<699::AIDJCLP2270500505>3.0.CO;2-C

Ingram, R. E. (1990). Self-focused attention in clinical disorders: Review and a conceptual model. Psychological Bulletin, 107, 156-176. doi: 10.1037/0033-2909.107.2.156

John, O., Donahue, E., \& Kentle, R. (1991). The "Big Five" InventoryVersions $4 a$ and 54. Berkeley: University of California, Berkeley.

Larsen, R. J., Diener, E., \& Emmons, R. A. (1986). Affect intensity and reactions to daily life events. Journal of Personality and Social Psychology, 51, 803-814. doi:10.1037/0022-3514.51.4.803

Malouff, J. M., Thorsteinsson, E. B., \& Schutte, N. S. (2005). The relationship between the five-factor model of personality and symptoms of clinical disorders: A meta-analysis. Journal of Psychopathology and Behavioral Assessment, 27, 101-114. doi:10.1007/s10862-005-5384-y

McAdams, D. P. (1996). Personality, modernity and the storied self: A contemporary framework for studying persons. Psychological Inquiry, 7 , 295-321. doi:10.1207/s15327965pli0704_1

McAdams, D. P., Josselson, R., \& Lieblich, A. (Eds.). (2006). Identity and story: Creating self in narrative. Washington, DC: American Psychological Association. doi:10.1037/11414-000

McAdams, D. P., \& McLean, K. C. (2013). Narrative identity. Current Directions in Psychological Science, 22, 233-238. doi:10.1177/ 0963721413475622 
McClelland, G. H., \& Judd, C. M. (1993). Statistical difficulties of detecting interactions and moderator effects. Psychological Bulletin, 114, 376-390. doi:10.1037/0033-2909.114.2.376

Mor, N., \& Winquist, J. (2002). Self-focused attention and negative affect: A meta-analysis. Psychological Bulletin, 128, 638-662. doi:10.1037/ 0033-2909.128.4.638

Newby, J. M., \& Moulds, M. L. (2011). Intrusive memories of negative events in depression: Is the centrality of the event important? Journal of Behavior Therapy and Experimental Psychiatry, 42, 277-283. doi: 10.1016/j.jbtep.2010.12.011

Neyer, F. J., \& Lehnart, J. (2007). Relationships matter in personality development: Evidence from an 8-year longitudinal study across young adulthood. Journal of Personality, 75, 535-568. doi:10.1111/j.14676494.2007.00448.x

Ogle, C. M., Rubin, D. C., \& Siegler, I. C. (in press). Changes in neuroticism following trauma exposure. Journal of Personality.

Ormel, J., Rosmalen, J., \& Farmer, A. (2004). Neuroticism: A noninformative marker of vulnerability to psychopathology. Social Psychiatry and Psychiatric Epidemiology, 39, 906-912. doi:10.1007/s00127004-0873-y

Ozer, E. J., Best, S. R., Lipsey, T. L., \& Weiss, D. S. (2003). Predictors of posttraumatic stress disorder and symptoms in adults: A meta-analysis. Psychological Bulletin, 129, 52-73. doi:10.1037/0033-2909.129.1.52

Perri, L. M., \& Keefe. F. J. (2008). Applying centrality of event to persistent pain: A preliminary view. Journal of Pain, 9, 265-271. doi:10.1016/j.jpain.2007.10.019

Pinto-Gouveia, J., \& Matos, M. (2011). Can shame memories become a key to identity? The centrality of shame memories. Applied Cognitive Psychology, 25, 281-290. doi:10.1002/acp.1689

Resick, P. A., \& Schnicke, M. K. (1992). Cognitive processing therapy for sexual assault victims. Journal of Consulting and Clinical Psychology, 60, 748-756. doi:10.1037/0022-006X.60.5.748

Roberts, B. W., Walton, K. E., \& Viechtbauer, W. (2006). Patterns of mean-level change in personality traits across the life course: A metaanalysis of longitudinal studies. Psychological Bulletin, 132, 1-25. doi: 10.1037/0033-2909.132.1.1

Robinaugh, D. J., \& McNally, R. J. (2010). Autobiographical memory for shame and guilt provoking events: Association with psychological symptoms. Behaviour Research and Therapy, 48, 646-652. doi: 10.1016/j.brat.2010.03.017

Rogers, W. M. (2002). Theoretical and mathematical constraints of interactive regression models. Organizational Research Methods, 5, 212230.

Rubin, D. C. (2006). The basic-systems model of episodic memory, Perspectives on Psychological Science, 1, 277-311. doi:10.1111/j.17456916.2006.00017.x

Rubin, D. C. (2011). The coherence of memories for trauma: Evidence from posttraumatic stress disorder. Consciousness and Cognition, 20, 857-865. doi:10.1016/j.concog.2010.03.018

Rubin, D. C., Berntsen, D., \& Bohni, M. K. (2008). A memory-based model of posttraumatic stress disorder: Evaluating basic assumptions underlying the PTSD diagnosis. Psychological Review, 115, 985-1011. doi:10.1037/a0013397

Rubin, D. C., Boals, A., \& Berntsen, D. (2008). Memory in posttraumatic stress disorder: Properties of voluntary and involuntary, traumatic and nontraumatic autobiographical memories in people with and without posttraumatic stress disorder symptoms. Journal of Experimental Psychology: General, 137, 591-614. doi:10.1037/a0013165

Rubin, D. C., Dennis, M. F., \& Beckham, J. C. (2011). Autobiographical memory for stressful events: The role of autobiographical memory in posttraumatic stress disorder. Consciousness and Cognition, 20, 840856. doi:10.1016/j.concog.2011.03.015
Rubin, D. C., \& Feeling, N. (2013). Measuring the severity of negative and traumatic events. Clinical Psychological Science. Advance online publication. doi:10.1177/2167702613483112

Rubin, D. C., Hoyle, R. H., \& Leary, M. R. (2012). Differential predictability of four dimensions of affect intensity. Cognition \& Emotion, 26, 25-41. doi:10.1080/02699931.2011.561564

Rush, A. J., Trivedi, M. H., Ibrahim, H. M., Carmody, T. J., Arnow, B., Klein, D. N., . . Keller, M. B. (2003). The 16-item Quick Inventory of Depressive Symptomatology (QIDS), clinician rating (QIDS-C), and self-report (QIDS-SR): A psychometric evaluation in patients with chronic major depression. Biological Psychiatry, 54, 573-583. doi 10.1016/S0006-3223(02)01866-8

Schuettler, D., \& Boals, A. (2011). The path to posttraumatic growth versus PTSD: Contributions of event centrality and coping. Journal of Loss and Trauma, 16, 180-194. doi:10.1080/15325024.2010.519273

Specht, J., Egloff, B., \& Schmukle, S. C. (2011). Stability and change of personality across the life course: The impact of age and major life events on mean-level and rank order stability of the Big Five. Journal of Personality and Social Psychology, 101, 862-882. doi:10.1037/ a0024950

Spence, D. P. (1982). Narrative truth and historical truth. New York, NY Norton.

Spitzer, R. L., Kroenke, K., Williams, J. B. W., \& Lowe, B. (2006). A brief measure for assessing generalized anxiety disorder. Archives of Internal Medicine, 166, 1092-1097. doi:10.1001/archinte.166.10.1092

Sutin, A. R., Costa, P. T., Wethington, E., \& Eaton, W. (2010). Turning points and lessons learned: Stressful life events and personality trait development across middle adulthood. Psychology and Aging, 25, 524533. doi:10.1037/a0018751

Teasdale, J. D., \& Green, H. A. C. (2004). Ruminative self-focus and autobiographical memory. Personality and Individual Differences, 36, 1933-1943. doi:10.1016/j.paid.2003.08.022

Tedeschi, R. G., \& Calhoun, L. G. (1996). The Posttraumatic Growth Inventory: Measure the positive legacy of trauma. Journal of Traumatic Stress, 9, 455-471. doi:10.1002/jts.2490090305

Tellegen, A. (1985). Structures of mood and personality and their relevance to assessing anxiety, with an emphasis on self-report. In A. H. Tuma \& J. D. Maser (Eds.), Anxiety and the anxiety disorders (pp. 681-706). Hillsdale, NJ: Erlbaum.

Watson, D., \& Clark, L. A. (1984). Negative affectivity: The disposition to experience negative aversive emotional states. Psychological Bulletin 96, 465-490. doi:10.1037/0033-2909.96.3.465

Watson, D., Clark, L. A., \& Tellegen, A. (1988). Development and validation of brief measures of positive and negative affect: The PANAS scales. Journal of Personality and Social Psychology, 54, 1063-1070. doi:10.1037/0022-3514.54.6.1063

Weathers, F. W., Litz, B. T., Huska, J. A., \& Keane, T. M. (1994). The PTSD checklist $(P C L)$. Unpublished scale available from the National Center for PTSD.

Wegner, D. M., \& Zanakos, S. (1994). Chronic thought suppression. Journal of Personality, 62, 615-640. doi:10.1111/j.1467-6494.1994 tb00311.x

Weiss, D. S., \& Marmar, C. R. (1997). The Impact of Event ScaleRevised. In J. P. Wilson \& T. M. Keane (Eds.), Assessing psychological trauma and PTSD: A practitioner's handbook (pp. 399-411). New York, NY: Guilford Press.

Whisman, M. A., \& McClelland, G. H. (2005). Designing, testing, and interpreting interactions and moderator effects in family research. Jour nal of Family Psychology, 19, 111-120. doi:10.1037/0893-3200.19.1 .111

Received August 1, 2013

Revision received October 16, 2013

Accepted October 19, 2013 